\title{
Painful and painless myocardial ischemia detected by elevated level of high-sensitive troponin in patients with hypertrophic cardiomyopathy
}

\author{
Adam Gębka ${ }^{1}$, Renata Rajtar-Salwa ${ }^{1}$, Artur Dziewierz ${ }^{2}$, Paweł Petkow-Dimitrow ${ }^{2}$ \\ ${ }^{1}$ Second Department of Cardiology and Cardiovascular Interventions, University Hospital, Krakow, Poland \\ ${ }^{2}$ Second Department of Cardiology, Jagiellonian University Medical College, Krakow, Poland
}

Adv Interv Cardiol 2018; 14, 2 (52): 195-198

DOI: https://doi.org/10.5114/aic.2018.76413

\section{Introduction}

High-sensitivity troponin I (hs-Tnl), an extra-precise biomarker for the detection of even small myocardial injury caused by ischemia, has been successfully used in patients with hypertrophic cardiomyopathy $(\mathrm{HCM})[1,2]$. In the most recent studies $[3,4]$, measurements of hs-Tnl levels were synchronized with a noninvasive assessment of hemodynamic parameters in the following way: first, resting echocardiography (including a provocative maneuver to induce a left ventricular outflow tract (LVOT) gradient) was performed, followed by ambulatory electrocardiography (ECG) Holter monitoring with devices which allow potential episodes of angina pectoris to be marked by the patients. After the 24-hour ECG Holter monitoring in conditions of typical everyday physical activity, the measurement of hsTnl level was immediately performed. Interestingly, the biomarker level had a close time relationship with findings on Holter monitoring [3] and echocardiography [4].

High-sensitivity troponin I levels were associated with both an increased heart rate during Holter monitoring [3] and a provoked LVOT gradient [4] (stimuli provoking myocardial ischemia). These findings are corroborated by previous studies in invasive and nonphysiological, atrial pacing stressors [5-7].

From the technical point of view, in patients with $\mathrm{HCM}$, verification of angina pectoris by resting or exercise ECG is practically impossible because of common abnormalities visible on resting ECG (with ischemic-like changes or significant deformation of the QT complex).

\section{Aim}

Nowadays, hs-Tn level measurement seems to be ideal for precise verification of myocardial injury due to the occurrence of ischemia in patients with HCM. The aim of this study was to collect information about episodes of angina pectoris occurring in the 24-hour period preceding the hs-Tnl level measurement, both in outpatients from a clinic and patients hospitalized due to cardiac signs and symptoms.

\section{Material and methods}

A group of 100 consecutive patients with HCM, both from ambulatory care and admitted to the clinic due to cardiac signs and symptoms (pooled group), were recruited to the study. Patients from our previous ambulatory studies were included in the current study; however, the present investigation is different from the previous one $[3,4]$ and is based on the history of angina pectoris within $24 \mathrm{~h}$ before the hs-Tnl measurement. We attempted to investigate a full spectrum of patients from asymptomatic ones to those with severe symptoms requiring hospitalization. Most patients received pharmacotherapy (Table I).

The exclusion criteria were as follows: (a) ST-segment or non-ST-segment elevation myocardial infarction (current or previous), (b) previous septal alcohol ablation for LVOT gradient reduction, (c) significant coronary stenosis on coronary angiography or (d) renal failure, (e) diabetes mellitus, and (f) regular sports activity. We used criteria a, b, c to exclude patients with concomitant atherosclerotic stenosis/occlusion of epicardial coronary arteries. We wanted to include only patients with small vessel disease, which is a common abnormality in HCM at any age. In such pathology, ischemia may be induced by coronary microvasculature abnormalities superimposing on other provoking factors - massive LV hypertrophy, LVOT gradi-

\section{Corresponding author:}

Paweł Petkow-Dimitrow MD, PhD, Second Department of Cardiology, Jagiellonian University Medical College, 17 Kopernika St, $31-501$ Krakow, Poland, phone: +48 1242471 70, e-mail: dimitrow@mp.pl

Received: 7.03.2018, accepted: 11.04.2018. 
Table I. Baseline characteristics $(n=73)$

\begin{tabular}{|c|c|}
\hline Variable & Value \\
\hline Ejection fraction, mean \pm SD (\%) & $62.4 \pm 9.2$ \\
\hline Maximum LV thickness, mean \pm SD $[\mathrm{mm}]$ & $21.4 \pm 4.8$ \\
\hline Resting LVOT gradient, mean $\pm \mathrm{SD}[\mathrm{mm} \mathrm{Hg}]$ & $27.11 \pm 16.53$ \\
\hline Resting LVOT gradient, > 30 mm Hg, $n$ (\%) & $20(27.4)$ \\
\hline Left atrial diameter, mean \pm SD $[\mathrm{mm}]$ & $48.7 \pm 10.2$ \\
\hline LV end-diastolic diameter, mean \pm SD [mm] & $42.5 \pm 7.9$ \\
\hline $\begin{array}{l}\text { Episode of angina pectoris during } 24 \mathrm{~h} \\
\text { before } \mathrm{hs} \text {-Tnl measurement (ambulatory, } \\
\text { hospitalization), } n(\%)\end{array}$ & $20(27.4)$ \\
\hline Dyspnea at any time, $n(\%)$ & $39(53.2)$ \\
\hline Syncope at any time, $n(\%)$ & $18(24.6)$ \\
\hline NSVT at any time, $n(\%)$ & $24(32.8)$ \\
\hline Sudden death in family history, $n(\%)$ & $19(26)$ \\
\hline Creatinine, mean $\pm \mathrm{SD}[\mu \mathrm{g} / \mathrm{l}]$ & $89.1 \pm 12.5$ \\
\hline \multicolumn{2}{|c|}{ Drugs used during analyzed 24-hour period, $n$ (\%): } \\
\hline$\beta$-blocker (metoprolol, bisoprolol, sotalol) & $54(74)$ \\
\hline Verapamil & $16(22)$ \\
\hline Diuretics & $6(8)$ \\
\hline None & $4(5)$ \\
\hline
\end{tabular}

hs-Tnl - high-sensitivity troponin I, LVOT - left ventricular outflow tract, LV - left ventricular, NSVT - nonsustained ventricular tachycardia.

ent, tachycardia. Renal failure is a common extra-cardiac factor responsible for Tnl elevation. Regular sports activity may induce repetitive ischemia in the predisposed patients. This issue requires further investigation. Diabetes mellitus may be associated with silent ischemia from epicardial coronary arteries (necessity of exclusion of painless macrovascular stenosis).

The final sample included 73 patients with HCM (age: $42 \pm 10$ years; 40 men and 33 women). Ambulatory patients were asked to maintain normal physical activity during the 24-hour period before the hs-Tnl level measurement. In hospitalized patients, the history of cardiac symptoms in the 24-hour period before admission was taken, and, finally, the hs-Tnl level was measured at admission. The cut-off value $19 \mathrm{ng} / \mathrm{l}$ was used according to the producer's instructions (bioMerieux VIDAS High sensitive Troponin I). The $99^{\text {th }}$ percentile of a presumably healthy population, the recommended cut-off has been defined at $19 \mathrm{ng} / \mathrm{l}$.

Patients were divided into 2 groups: hs-Tnl-positive and hs-Tnl-negative. In the next stage, the group with the elevated level of hs-Tnl was divided into 2 further subgroups: one with angina pectoris (painful ischemia $(\mathrm{AP}+))$ and the other without angina pectoris (painless, silent ischemia (AP-)). The study protocol was approved by a local institutional review board.

\section{Statistical analysis}

Continuous variables were presented as mean (SD) or median (interquartile range - IQR). The levels of hs-Tnl between AP+ and AP- were compared by the Mann-Whitney test. The majority of echocardiographic parameters with normal distribution (according to the Kolmogorov-Smirnov test) were compared using Student's t-test. For comparison of one echocardiographic parameter the Mann-Whitney test was used. A $p$-value of $<0.05$ was considered statistically significant.

\section{Results}

Baseline characteristics of 73 patients are presented in Table I.

Hs-Tnl was detected in all patients (range: 1.5-40,000 $\mathrm{ng} / \mathrm{l})$. Increased levels were revealed in 35 patients (troponin-positive group), and "normal-low" levels in 38 patients (troponin-negative group). A total of 17 patients from the troponin-positive subgroup had perceptible angina pectoris $(\mathrm{AP}+)$ and the remaining 18 patients were without angina pectoris (AP-). The level of troponin was significantly higher in the AP+ subgroup in comparison with the AP- subgroup (median, IQR: $100.2 \mathrm{ng} / \mathrm{l}, 60.1-$ $1640.0 \mathrm{ng} / \mathrm{l}$ vs. $36.4 \mathrm{ng} / \mathrm{l}, 20.5-70.1 \mathrm{ng} / \mathrm{l} ; p=0.027)$. In the troponin-negative subgroup, 3 out of 38 patients (7.7\%) had a short episode of mild angina pectoris in the first hours of the 24-hour period (the measurement of hs-Tnl levels was probably performed after the peak value in a decreasing phase of the time profile of hs-Tnl release).

There were no differences in echocardiographic parameters between $\mathrm{AP}-$ and $\mathrm{AP}+$ subgroups (only echocardiographic parameters were synchronously measured with hs-TnI) (Table II).

\section{Discussion}

According to the third universal definition of myocardial infarction from 2012, a (nearly) normal coronary angiogram does not exclude acute coronary syndrome [8]. The guidelines defined the mechanism of type 2 myocardial infarction as secondary to ischemic imbalance causing myocardial injury with necrosis where conditions other than coronary contribute to the imbalance between myocardial oxygen supply and/or demand. In $\mathrm{HCM}$, the potential mechanism of myocardial ischemia includes: hypertrophied myocardium, coronary microvascular disease, tachycardia, and increased LVOT gradient. In our group, almost $50 \%$ of the patients had a positive hs-Tnl test result, out of which approximately $25 \%$ had silent ischemia and $25 \%$ had painful angina pectoris (in this group, the median value of hs-Tnl was elevated $>5 \times$ normal value, i.e. a significant level). These values 
Table II. Comparison of echocardiographic parameters recorded no later than $24 \mathrm{~h}$ after hs-Tnl measurement between patients without versus with angina pectoris

\begin{tabular}{lccc} 
Parameter & hs-Tnl (+) and AP- & hs-Tnl (+) and AP+ & $P$-value \\
\hline Ejection fraction, mean \pm SD (\%) & $63.0(7.1)$ & $61.2(14.2)$ & 0.63 \\
\hline LVOTG rest, median (interquartile range) $[\mathrm{mm} \mathrm{Hg}]$ & $19(7-50)$ & $16(6.5-61)$ & 0.88 \\
\hline LVOTG provocable, mean \pm SD $[\mathrm{mm} \mathrm{Hg}]$ & $41.1 \pm 39.0$ & $58.9 \pm 49.9$ & 0.24 \\
\hline Maximum LV thickness, mean \pm SD [mm] & $2.24 \pm 0.5$ & $2.19 \pm 0.48$ & 0.76 \\
\hline LV end-diastolic diameter, mean \pm SD [mm] & $4.38 \pm 0.81$ & $4.46 \pm 0.59$ & 0.74 \\
\hline Left atrial diameter, mean \pm SD [mm] & $5.02 \pm 0.97$ & $4.85 \pm 0.80$ & 0.58
\end{tabular}

hs-TnI - high-sensitivity troponin I, AP - angina pectoris, LVOTG - left ventricular outflow tract gradient, LV - left ventricular.

are alarming and important. Recently, it has been proposed that stress echocardiography has a significant prognostic role in patients with $\mathrm{HCM}$, with ischemic endpoints showing a greater predictive accuracy than hemodynamic ones [9].

Until 2017, both United States and European guidelines for exercise and sports participation in patients with HCM had issued consistent recommendations against participation in all kinds of low-intensity competitive sports and even advised not to undertake vigorous activities on a recreational basis. However, recently, exercises such as long-term training, fitness, and sports activity were reported as beneficial in patients with HCM $[10,11]$.

Based on the association between the elevated troponin level in patients with HCM and (i) tachycardia, (ii) nonsustained ventricular tachyarrhythmia (NSVT) [3], (iii) resting and provoked LVOT gradients [4], (iv) high risk for sudden cardiac death [12] and symptomatic angina pectoris (described in this study), we may suggest that any exercise in these patients (performed either for training or diagnostic purposes) should be monitored by frequent troponin level measurements after the exercise test (4, 8, 12 and $24 \mathrm{~h}$ after exercise). The proposed time profile of troponin sampling has been partly based on a very recent publication in a highly selected group of preclinical HCM patients [13] where after exercise the first and only hs-Tnl measurement was performed $4 \mathrm{~h}$ after stress. We propose a more detailed time profile to detect peak value hs-Tnl after exercise.

The most important subgroup is that of patients with silent myocardial ischemia (approximately 25\%), who should be discouraged from training (due to lack of awareness of induction of ischemia).

Our study has several limitations. First, the number of included patients was limited by several exclusion criteria. Next, the current pharmacological treatment was maintained; particularly $\beta$-blockers were not withdrawn in ambulatory patients as our preliminary study showed that $\beta$-blocker withdrawal might not be safe in this group of patients.
The next limitation is the fact that only echocardiographic parameters were synchronously measured with hs-Tnl. Another limitation is the evidence that although the major mechanism of troponin release is ischemia with myocardial injury there are several other mechanisms, as reported by White [14]. Finally, two more limitations are the lack of imaging both for stress test induced ischemia and stress resulting in ischemic injury (i.e. late enhancement in cardiac magnetic imaging reported by Grommans and Cramer) [15]. In this preliminary study, we aimed to assess hs-Tnl (frequency of painful/painless ischemia) only in patients not exposed to unnatural stress conditions (for logistic and ethical reasons). Previously we observed the release of hs-Tnl after natural, spontaneous stressors such as supraventricular [16] and ventricular tachycardia [17].

\section{Conclusions}

We confirmed that the assessment of the hs-Tnl level is useful to monitor a full spectrum of patients with HCM (from asymptomatic patients to those with severe symptoms). Approximately $25 \%$ of the patients had angina pectoris with significantly elevated hs-Tnl levels, and approximately $25 \%$ had silent myocardial ischemia with slightly/moderately elevated hs-Tnl levels. The echocardiographic parameters did not differ between the painful and painless subgroup of patients.

From a clinical perspective, these findings suggest the need to improve pharmacological/nonpharmacological management of patients with HCM and discourage systematic physical activity in the troponin-positive subgroup (considering the third universal definition of myocardial infarction).

\section{Conflict of interest}

The authors declare no conflict of interest.

\section{References}

1. Kubo T, Kitaoka H, Yamanaka S, et al. Significance of high-sensitivity cardiac troponin $\mathrm{T}$ in hypertrophic cardiomyopathy. J Am Coll Cardiol 2013; 62: 1252-9. 
2. Kawasaki T, Sakai C, Harimoto K, et al. Usefulness of high-sensitivity cardiac troponin and brain natriuretic peptide as biomarkers of myocardial fibrosis in patients with hypertrophic cardiomyopathy. Am J Cardiol 2013; 112: 867-2.

3. Htadij R, Rajtar-Salwa R, Dimitrow PP. Associaton of elevated troponin levels with increased heart rate and higher frequency of nonsustained ventricular tachycardia in hypertrophic cardiomyopathy. Pol Arch Intern Med 2017; 126: 445-7.

4. Hładij R, Rajtar-Salwa R, Dimitrow PP. Troponin as ischemic biomarker is related with all three echocardiographic risk factors for sudden death in hypertrophic cardiomyopathy (ESC Guidelines 2014). Cardiovasc Ultrasound 2017; 15: 24.

5. Cannon RO 3rd, Rosing DR, Maron BJ, et al. Myocardial ischemia in patients with hypertrophic cardiomyopathy: contribution of inadequate vasodilator reserve and elevated left ventricular filling pressures. Circulation 1985; 71: 234-43.

6. Cannon RO 3rd, Schenke WH, Maron BJ, et al. Differences in coronary flow and myocardial metabolism at rest and during pacing between patients with obstructive and patients with nonobstructive hypertrophic cardiomyopathy. J Am Coll Cardiol 1987; 10: 53-62.

7. Cannon RO 3rd, Dilsizian V, O'Gara PT, et al. Myocardial metabolic, hemodynamic, and electrocardiographic significance of reversible thallium-201 abnormalities in hypertrophic cardiomyopathy. Circulation 1991; 83: 1660-7.

8. Thygesen K, Alpert JS, Jaffe AS, et al. Third universal definition of myocardial infarction. Eur Heart J 2012; 33: 2551-67.

9. Ciampi Q, Olivotto I, Gardini C, et al. Prognostic role of stress echocardiography in hypertrophic cardiomyopathy: the International Stress Echo Registry. Int J Cardiol 2016; 219: 331-8.

10. Dejgaard LA, Haland TF, Lie $\mathrm{OH}$, et al. Vigorous exercise in patients with hypertrophic cardiomyopathy. Int J Cardiol 2018; 250: 157-63.

11. Saberi S, Wheeler M, Bragg-Gresham J, et al. Effect of moderate-intensity exercise training on peak oxygen consumption in patients with hypertrophic cardiomyopathy: a randomized clinical trial. JAMA 2017; 317: 1349-57.

12. Rajtar-Salwa R, Hładij R, Dimitrow PP. Elevated level of troponin but not $\mathrm{N}$-terminal-pro-brain natriuretic peptide is associated with increased risk of sudden cardiac death in hypertrophic cardiomyopathy calculated according to ESC Guidelines 2014. Dis Markers 2017; 2017: 9417908.

13. Ho JE, Shi L, Day SM, et al. Biomarkers of cardiovascular stress and fibrosis in preclinical hypertrophic cardiomyopathy. Open Heart 2017; 4: e000615.

14. White HD. Pathobiology of troponin elevations: do elevations occur with myocardial ischemia as well as necrosis? J Am Coll Cardiol 2011; 57: 2406-8.

15. Gommans DF, Cramer GE, Bakker J, et al. High T2-weighted signal intensity is associated with elevated troponin $\mathrm{T}$ in hypertrophic cardiomyopathy. Heart 2017; 103: 293-9.

16. Rajtar-Salwa R, Dimitrow PP, Miszalski-Jamka T. Role of cardiac magnetic resonance in differentiating between acute coronary syndrome and apical hypertrophic cardiomyopathy. Adv Interv Cardiol 2016; 12: 380-2.

17. Hładij R, Rajtar-Salwa R, Dziewierz A, Dimitrow PP. The diagnostic role of cardiac magnetic resonance used "first and last time in life" in a patient with a suspected dilated phase of hypertrophic cardiomyopathy. Adv Interv Cardiol 2017; 13: 178-9. 